\title{
Can satellite dwarfs excite galactic warps?
}

\author{
Jeremy Bailin ${ }^{1}$ and Matthias Steinmetz ${ }^{2}$ \\ ${ }^{1}$ Centre for Astrophysics and Supercomputing, Swinburne University of Technology, Mail H39, \\ PO Box 218, Hawthorn, Victoria, 3122, Australia; email: jbailin@astro.swin.edu.au \\ ${ }^{2}$ Astrophysikalisches Institut Potsdam, An der Sternwarte 16, D-14482, Potsdam, Germany; \\ email: msteinmetz@aip.de
}

\begin{abstract}
We present a series of $N$-body simulations examining the tidal impact of satellite dwarfs on galactic disks, in particular on the abilities of satellites to excite warps like the one seen in the disk of the Milky Way. We have examined satellites with masses spanning 1.5 orders of magnitude, ranging from the Sgr dSph to the LMC. Orbits similar to the eccentric polar orbit of Sgr were examined, along with orbits of intermediate inclination and of lower eccentricity. The fundamental physical processes which govern the transfer of angular momentum between the satellite and disk, and within the disk, are identified to be precession of the satellite orbit, shrinking of the satellite orbit due to dynamical friction, warping of the disk within a slewing potential, and differential precession. We find that although very large satellites can tidally excite warps shortly after each perigalactic passage, the effects of such satellites are quite destructive and the resulting disks have smaller warps and appear more disturbed than the disks of the Milky Way and other strongly-warped galaxies. Smaller satellites, however, may be capable of producing the minor warps typical of many disk galaxies.
\end{abstract}

Keywords. stellar dynamics, methods: n-body simulations, Galaxy: disk, galaxies: kinematics and dynamics, galaxies: dwarf, galaxies: interactions, galaxies: individual (Sgr dSph)

\section{Introduction}

The disk of the Milky Way is warped like an integral sign, rising above the plane on one side and falling below the plane on the other. This warp is seen both in maps of neutral hydrogen (e.g., Diplas \& Savage(1991)) and in the stellar distribution (e.g., Drimmel et al.(2000)). The Milky Way is far from unique in this regard; the planes of most external galaxies are warped to some degree (Reshetnikov \& Combes(1998), Schwarzkopf \& Dettmar(2001), García-Ruiz et al.(2002b)). While many of these warps are of the dramatic variety normally envisioned by the phrase "warped galaxy" and exemplified by the Milky Way, $1 / 3$ of warps are of a much more minor variety, with warp angles less than $5^{\circ}$ (García-Ruiz et al.(2002b)).

The ubiquity of warped galaxies poses a puzzle. Isolated warps have a strong tendency to damp and disperse due to the differential precession of the disk (Kahn \& Woltjer(1959), Hunter \& Toomre(1969)). There are two possible solutions to this puzzle: either some phenomenon stabilizes warps against differential precession, or warps are dynamically transitory but are excited so often that the probability of observing any particular galaxy in a warped state is high. Many authors have investigated the first possibility; most of these studies rely on either the galactic dark matter halo (Sparke \& Casertano(1988), Kuijken(1991), Debattista \& Sellwood(1999), Ideta et al.(2000)) or infalling intergalactic gas (Kahn \& Woltjer(1959), López-Corredoira et al.(2002)) to stabilize the warp. In this contribution, we investigate the second possibility, that there exists a method of easily exciting warps. 
The particular perturbations whose effects we examine are the dwarf satellites surrounding disk galaxies. Ideally, a description of warped galaxies should at least be able to explain the Milky Way warp, about which we have more information than about any other galaxy. While the Magellanic Clouds have often been proposed as the perturbation responsible for the Milky Way's warp (Weinberg(1998), Tsuchiya(2002)), García-Ruiz et al.(2002a) demonstrated that a warp caused by the Magellanic Clouds would have its line of nodes oriented orthogonal to the observed line of nodes. Therefore, the Magellanic Clouds are not suitable candidates for producing the Milky Way warp.

The orbital plane of the Sagittarius dwarf spheroid galaxy, on the other hand, is oriented correctly with respect to the Galactic warp. It has a small perigalactic distance, resulting in a very strong tidal torque. The radius of its paragalacticon matches where the warp is strongest. Moreover, there is a remarkable coincidence between the orbital angular momentum of Sagittarius and the misaligned angular momentum in the disk responsible for the warp, which suggests that the systems are coupled, i.e. that Sagittarius is responsible for the warp (Bailin(2003)).

Simulations of satellite-disk interactions that have been performed to date cannot properly test this hypothesis. Tsuchiya(2002) tested the warping effects of the Magellanic Clouds, but did not investigate a satellite with the properties of Sgr, while Ibata \& Razoumov(1998) studied the effects of Sgr assuming it had a mass of $5 \times 10^{9} M_{\odot}$, much larger than current estimates of $(2-5) \times 10^{8} M_{\odot}$ (Law et al.(2005)), and did not include a live Galactic halo, which may amplify the torque of the satellite (Weinberg(1998)).

We have performed a set of $N$-body simulations designed to study the interactions between satellites and warps, and in particular to test the hypothesis that the Sgr dSph galaxy is responsible for the warp of the Milky Way.

\section{The Simulations}

The simulations were performed using the parallel version of the GADGET $N$-body code (Springel et al.(2001)), and only take into account gravitational forces. The disk galaxy model is based on the Milky Way models of Dehnen \& Binney(1998), particularly model 3. It consists of 1048576 halo particles, 969438 disk particles and 79138 bulge particles. The satellite consists of a single particle containing the entire satellite mass. We investigated the effects of a satellite with the estimated mass of Sgr, $4 \times 10^{8} M_{\odot}$, along with more massive satellites with masses of $2 \times 10^{9} M_{\odot}$ and $1 \times 10^{10} M_{\odot}$ respectively in order to better understand how the satellite mass affects the strength of the coupling between satellites and the warp.

Each simulation was evolved from $t=0$ to $t=4$ Gyr. We have adopted a very conservative timestep with parameter $\eta=0.01$ to ensure that vertical oscillations were not artificially amplified by integration errors. This resulted in an average timestep of $8 \times 10^{-5}$ Gyr.

The initial orbital parameters were chosen to mimic those of Sgr: a polar orbit with an apogalacticon of $70 \mathrm{kpc}$ and a perigalacticon of $13 \mathrm{kpc}$ (Helmi \& White(2001)). However, a wide range of additional orbits were simulated in order to understand the effect of the orbital eccentricity and inclination.

Because the satellite is represented by a single particle, its internal degrees of freedom are not taken into account. The internal rotation of the satellite cannot be an important reservoir of angular momentum in the system unless the satellite is disrupted; therefore, our results are expected to be valid during the time before which a fully self-consistent satellite would disrupt or lose most of its mass, i.e. for a couple of orbits. 

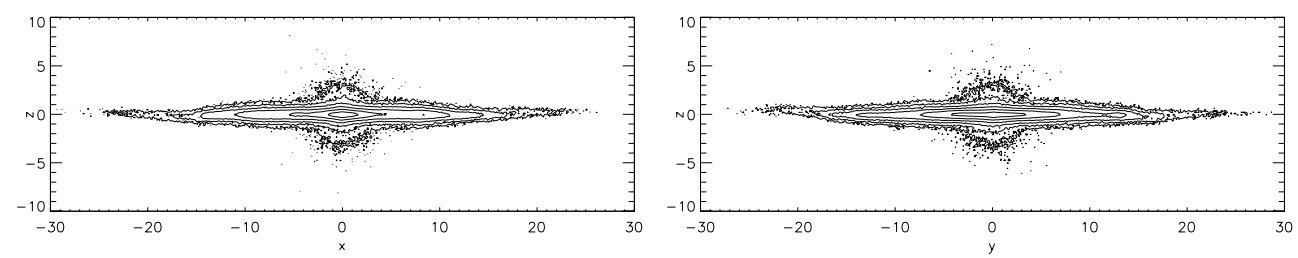

Figure 1. Log-spaced contours of the projected density of the bulge and disk at the time of maximum warp (250 Myr after the perigalactic passage) for a satellite of mass $2 \times 10^{9} M_{\odot}$ on a polar orbit. The left panel is the $x-z$ projection, while the right panel is the $y-z$ projection. The global tilt of the disk has been removed.
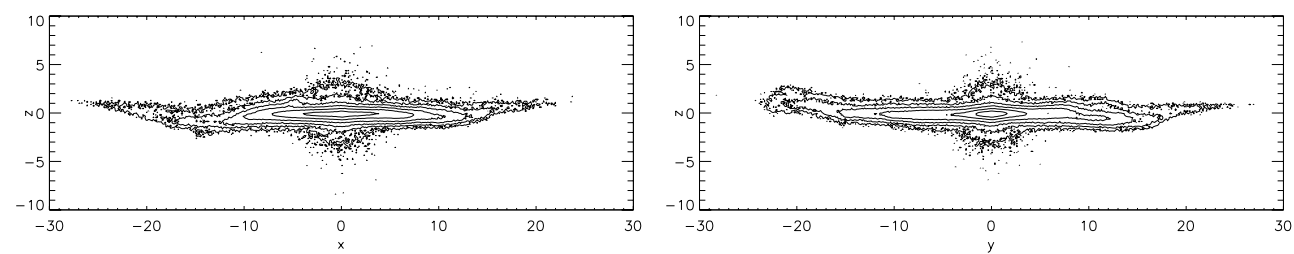

Figure 2. As in Figure 1, but for a satellite of mass $1 \times 10^{10} M_{\odot}$. The maximum warp occurs 200 Myr after the perigalactic passage.

\section{Results}

There are four fundamental processes that govern the internal angular momentum of the disk in the presence of the satellite. Firstly, the satellite precesses in the flattened potential of the disk. The change in the satellite's orbital angular momentum is balanced by a corresponding change in the angular momentum of the disk, causing it to tilt. Secondly, dynamical friction causes the satellite orbit to sink. This loss of angular momentum is transferred to the halo and to the disk, especially the outer disk, causing it to tilt and warp (Huang \& Carlberg(1997)). Thirdly, Ostriker \& Binney(1989) determined that if the potential of a disk slews, the angular momenta of rings within the disk tilt differentially toward the slewing vector, causing the disk to warp. Finally, differential precession in a warped disk quickly damps the warp in the absence of the above processes.

Our least massive satellite excites no visible warp in the galactic disk. Therefore, it appears that Sgr with its current mass cannot be responsible for the warp of the Milky Way. More massive satellites excites small warps in the disk. Figure 1 demonstrates the warp in the disk $250 \mathrm{Myr}$ after the perigalactic passage of our intermediate-mass satellite. The warp is barely visible at the left edge of the $y-z$ projection. Our most massive satellite excites a much stronger warp, as seen in Figure 2. This simulation corresponds to a satellite with the mass of the LMC on the orbit of Sgr. Even this warp is not as strong as the warp of the Milky Way, and moreover the disk appears much more disturbed than simply warped. Since it is unlikely that there is an unknown satellite of the Milky Way at least as massive as the LMC on an orbit at least as close as that of Sgr, the warp of the Milky Way is unlikely to be caused by an interaction with a satellite dwarf.

We find that the warp is strongest one disk rotation period after the perigalactic passage of the satellite. The warps are often asymmetric. We have performed simulations with satellites on orbits inclined $45^{\circ}$ to the disk, both on prograde and retrograde orbits, and found that the orbital orientation affects the orientation of the line of nodes of the warp, but does not have a strong effect on the warp amplitude. The angular momentum of the warp is typically $\approx 1 \%$ of the orbital angular momentum of the satellite. 


\section{Conclusions}

Very massive satellites are able to tidally excite small warps in disk galaxies. The warp reaches its maximum amplitude one disk rotation period after perigalacticon. The simulated warps are too small to explain strongly warped galaxies; in particular, the tidal torque of Sgr appears insufficient to cause the warp of the Milky Way. However, minor warps with amplitudes less than $5^{\circ}$ are typical of many disk galaxies, and large satellites are capable of exciting warps of this magnitude.

\section{References}

Bailin, J. 2003, ApJ 583, L79

Debattista, V.P. \& Sellwood, J.A. 1999, ApJ 513, L107

Dehnen, W. \& Binney, J. 1998, MNRAS 294, 429

Diplas, A. \& Savage, B.D. 1991, ApJ 377, 126

Drimmel, R., Smart, R.L. \& Lattanzi, M.G. 2000, A\& A 354, 67

García-Ruiz, I., Kuijken, K. \& Dubinski, J. 2002a, MNRAS 337, 459

García-Ruiz, I., Sancisi, R. \& Kuijken, K. 2002b, A\&A 394, 769

Helmi, A. \& White, S.D.M. 2001, MNRAS 323, 529

Huang, S. \& Carlberg, R.G. 1997, ApJ 480, 503

Hunter, C. \& Toomre, A. 1969, ApJ 155, 747

Ibata, R.A. \& Razoumov, A.O. 1998, A\&A 336, 130

Ideta, M., Hozumi, S., Tsuchiya, T. \& Takizawa, M. 2000, MNRAS 311, 733

Kahn, F.D. \& Woltjer, L. 1959, ApJ 130, 705

Kuijken, K. 1991, ApJ 376, 467

López-Corredoira, M., Betancort-Rijo, J. \& Beckman, J.E. 2002, A $\& A$ 386, 169

Law, D.R., Johnston, K.V. \& Majewski, S.R. 2005, ApJ 619, 807

Ostriker, E.C. \& Binney, J.J. 1989, MNRAS 237, 785

Reshetnikov, V. \& Combes, F. 1998, A\&A 337, 9

Schwarzkopf, U. \& Dettmar, R.-J. 2001, A\&A A 373, 402

Sparke, L.S. \& Casertano, S. 1988, MNRAS 234, 873

Springel, V., Yoshida, N. \& White, S.D.M. 2001, New Astronomy 6, 79

Tsuchiya, T. 2002, New Astronomy 7, 293

Weinberg, M.D. 1998, MNRAS 299, 499

\section{Discussion}

Martinez-Delgado: You have shown the prominent warp of NGC 5907. It is interesting to note that this galaxy has one of the brightest tidal streams ever discovered in an external galaxy. Do you think this is a coincidence?

BAILIN: No, probably not. The mass in the NGC 5907 stream is very large, probably more than Sgr. Also, the effects of a completely disrupted satellite, like in NGC 5907, may be different than with a single satellite.

DeBATtista: What is the velocity dispersion profile of your disks? In real galaxies warps are observed in gas which has a low $Q$. As $Q$ gets larger warps get smaller because gravitational stresses are spread over larger regions and lead to more coherent tilting than warping. I suspect that with a lower $Q$ in the outer parts you can get warps with smaller satellite mass.

BAILIN: We use $Q=1.5$. We find that if we reduce $Q$, the disk becomes quite unstable to bar formation, which induces $m=2$ vertical modes that dominate over the $m=1$ mode of the warp. 